\title{
6
}

\section{Enacting the Prevent Duty in Primary Schools}

\author{
Raquel da Silva, Giuditta Fontana, \\ and Megan A. Armstrong
}

\begin{abstract}
This chapter examines the enactment of the Prevent Duty in primary schools since its introduction in July 2015, with a focus on its broad implementation, the raising of Prevent-related concerns, and the

This chapter was supported by the British Academy Postdoctoral Fellowship (pf170092), the Leverhulme Early Career Fellowship (ECF-2015-026), and the Quality Outputs Scheme at the College of Social Sciences, University of Birmingham. We thank Hamdi Yasin, Rumaysa Mahmood, and Alice Russell for their research assistance. We thank Dr Charlotte Heath-Kelly and Dr Erzsebet Strausz for allowing us to adapt their questionnaire to this project.
\end{abstract}

R. da Silva $(\bowtie)$

University of Birmingham, Birmingham, UK

Centre for International Studies, ISCTE-IUL, Lisbon, Portugal

e-mail: R.B.P.daSilva@bham.ac.uk

G. Fontana

University of Birmingham, Birmingham, UK

e-mail: g.fontana@bham.ac.uk

M. A. Armstrong

Liverpool John Moores University, Liverpool, UK

e-mail: M.A.Armstrong@ljmu.ac.uk 
embedding of Prevent into fundamental British values (FBVs). Our findings illustrate how this policy enactment responds to a context determined by the idiosyncrasies of the primary school setting, of the wider community, and of the political and strategic environment. We find that primary school educators display a positive acceptance of the Prevent Duty, largely motivated by its incorporation in a wider professional culture of safeguarding and that, with rare exceptions, they are supportive of embedding Prevent in the teaching of FBVs.

Keywords Prevent Duty $\bullet$ Safeguarding $\bullet$ Education $\bullet$ Primary schools - Fundamental British values

In this chapter, we examine how the Prevent Duty has been enacted in primary schools since its introduction in July 2015. We focus on its broad implementation, the raising of Prevent-related concerns, and the embedding of Prevent into fundamental British values (FBVs).

Our findings illustrate how this policy enactment (Ball, McGuire, \& Braun, 2013) is dynamic, responding to a context determined by the idiosyncrasies of the primary school setting, as well as by developments in the wider community and political environment. Specifically, we find that the primary school educators who participated in this research displayed a positive acceptance of the Prevent Duty, largely motivated by its incorporation in a wider professional culture of safeguarding, and within existing pedagogical practices around teaching values. We also show how the implementation of the Duty is evolving away from focusing on Islamist extremism, under the pressure of a changing socio-political context. Furthermore, and in contrast with some of the other chapters in this volume, we encountered very few expressed criticisms either of the embedding of Prevent within the teaching of FBVs or indeed of the labelling of these values as 'British'.

Whilst this chapter does not aim to provide an exhaustive account of how the Prevent Duty is enacted in primary schools throughout the UK, the breadth and variety of our data allow us to offer a valuable snapshot of the main approaches adopted in the West Midlands and how educators have assessed the implications of these approaches for their local community. 


\section{Methods}

We draw on the findings of a larger project examining the operationalisation of the Prevent Duty in primary schools in the West Midlands, carried out in 2018-2019. The West Midlands is a particularly interesting case study of the implementation of the Prevent Duty because, at the time of writing, three of its six local authorities are Prevent priority areas: Birmingham, Coventry, and Walsall. Priority areas are geographic regions considered to have a higher threat level (Mastroe, 2016; Home Office, 2019). As such, they are provided with additional funding, a local Prevent coordinator, and in some cases one or more local Prevent Education Officers (PEOs) (see Chap. 2).

The project included an online survey, sent to all primary schools in the West Midlands. The survey was based on Charlotte Heath-Kelly and Erzsebet Strausz's survey on the Prevent Duty in the National Health Service (Heath-Kelly \& Strausz, 2019). Questions were added or rephrased to adapt the survey to the primary school context and to further explore scenarios related to right-wing extremism. Free-text boxes were added to afford participants the opportunity to reflect, comment, and elaborate on their responses. Between February and July 2019, 345 primary school educators answered the survey. This sample included educators at all stages of their career, teaching at all primary stages in state schools, academies, faith schools, and independent schools throughout the six local authorities in the West Midlands. The respondents included teaching assistants, teachers, and Designated Safeguarding Leads (DSLs) as well as school leaders.

The survey data was complemented by 32 semi-structured interviews with primary school educators in the West Midlands and five interviews with PEOs from the region. Questions focused on how primary school educators understood the Prevent Duty; how they enacted the Duty; and how they perceived the impact and implications of the Duty in their own sector of work. Throughout this chapter, we use both the free-text comments by survey respondents and excerpts from interview transcripts. 


\section{Implementing the Prevent Duty in Primary Education in the West Midlands}

The statutory guidance for keeping children safe in education (DfE, 2019) presents the Prevent Duty as part and parcel of wider safeguarding practices. Thus, schools, like all sectors affected (e.g., health, social services), are expected to integrate Prevent into existing safeguarding policies and procedures (Home Office, 2019). Primary and secondary schools specifically,

need to demonstrate that they are protecting children and young people from being drawn into terrorism by having robust safeguarding policies in place to identify children at risk, and intervening as appropriate. (Home Office, 2019)

Despite the controversy generated by the introduction of the Prevent Duty in sectors like education, and criticisms from some observers that the Duty leads to the 'blurring of social and security policy' (Ragazzi, 2017, p. 168), 74.8\% of survey respondents agreed or strongly agreed that 'The Prevent Duty belongs within education'. Some explained this view with reference to the belief that they are ideally positioned to notice and to deal with any behavioural changes, including signs of radicalisation:

teachers often get to know children very well and be able to see the changes in them to prevent radicalisation before it becomes too late. (Anonymous survey respondent)

In line with the existing literature on Preventing Violent Extremism (PVE) and education (Davies, 2016; Bryan, 2017; Busher, Choudhury, \& Thomas, 2019; Elwick \& Jerome, 2019), we found that the Prevent Duty, in the primary school context, has been broadly accepted as part and parcel of safeguarding. Indeed, the majority of survey respondents (71\%) agreed or strongly agreed with the statement 'The Prevent Duty is just safeguarding. It is the same as safeguarding pupils from domestic abuse and sexual abuse'. However, their responses to the Duty were not 
limited to a position of 'pragmatic acceptance' (Busher et al., 2019). Rather, some of the respondents adopted more positive attitudes, fuelled partly by the way that the Duty resonated with existing perceptions of young children as victims, as individuals at risk of abuse. One PEO elaborated on this point in an interview:

Actually, saying just like any other issue FGM, honour based violence, domestic abuse, all these different issues that can affect people, you see Prevent the same. So, what I say to them [primary school educators] is: when you are dealing with a CSE [child sexual exploitation] case you see the young people as victims, when you're dealing with a Prevent case you are doing the same thing, they're victims of radicalisation or grooming. (PEO1)

In the same line of thought, one survey respondent stated in the free-text comments:

As a school we must protect children from all forms of harm and radicalisation is a form of harm. (Anonymous survey respondent)

Some interviewees also underscored that aligning Prevent with safeguarding has helped educators come to terms with the policy as an instrument to protect rather than to police children and their families. As a headteacher put it, when seen in this light the Prevent Duty becomes:

just part of normal safeguarding, I don't want the big hysteria about it [...] I think it is just another part of safeguarding, in the same way that if you saw a child with a bruise, or if a child disclosed something to you. (E18, headteacher, Birmingham)

Similarly, another headteacher noted:

[if] there are still some headteachers that believe that Prevent is too invasive, erm, I'm not one of those I'm afraid. (E23, headteacher, Birmingham)

Only one survey respondent contemplated the potentially detrimental consequences of the Prevent Duty, in general, and of a Channel referral 
in particular, which he considered to be more than 'just safeguarding' as it 'can label people as terrorists' and 'lead to dreadful consequences that affect many people in the community'.

This overall confidence in the Prevent Duty and in its implementation is, as already mentioned, fuelled by its conflation with existing safeguarding policies and practices. According to our survey data, the quality of the Prevent-related training provided to educators has also played an important role here. The Department for Education requires that all staff undertake this type of training. The Home Office offers WRAP (Workshop to Raise Awareness about Prevent) training and a variety of external organisations have also developed Prevent-related training tailored to schools. This training should, according to the statutory guidance, give teachers 'the knowledge and confidence' to identify at-risk pupils, as well as how to refer pupils for further support (Home Office, 2019, paragraph 70). Among our survey respondents, 96\% stated that the training they had received explained the signs of radicalisation well or very well. This contributed to the overwhelming majority of survey respondents stating that they were confident or very confident in referring a query about radicalisation $(90.9 \%)$. As one of the respondents reported, they felt confident because they felt well equipped 'with the information needed to identify signs of radicalisation'.

The data collected through interviews was however less positive regarding the quality of the training received, particularly the WRAP training. In contrast to survey respondents, some interviewees emphasised that the Prevent training they attended was not suitable for the primary school context. Reflecting on the WRAP training, one interviewee stated:

I find it difficult to relate a lot of the training materials to this [primary school] environment because it is very much secondary based and teenager based and because of how the vulnerable groups are identified. In that respect you can't just walk away with the training materials and apply what you have learned. (E2, Assistant Head, Walsall)

In this sense, most interviewees suggested that the training ought to be better tailored to the age group they deal with by clearly presenting the risks and signs of radicalisation for primary school children, as they did 
not believe that they are the same as for secondary school pupils. Most of our primary school respondents saw children as potential victims rather than perpetrators when it came to Prevent-related issues. For example,

Generally [because of] the age of our children they probably are less susceptible to Prevent issues so we're not... if we get a trigger about a worry there is usually something else behind it. (E2, Assistant Head, Walsall)

This was not, however, the opinion of one of the PEOs interviewed, who considered that years 5 and 6 are 'crucial times when children could be radicalised' (PEO5), which justifies raising awareness, in the training context, of case studies of older children who are at risk of radicalisation.

In sum, the data collected through both surveys and interviews displayed a rather positive acceptance of the Prevent Duty, rooted in the incorporation of Prevent in a wider culture of safeguarding. Whilst survey respondents considered Prevent-related training had boosted their confidence in implementing the Duty, interviewees pointed out that training materials need to be better tailored to the primary school context.

\section{Raising Prevent-Related Safeguarding Concerns}

Despite their positive acceptance of the Prevent Duty, respondents were not passive in its implementation. In fact, we found that the enactment of the Duty was dynamic and responded to its wider environment. Respondent's reflections on the scenarios that would lead them to raise a Prevent-related safeguarding concern, illustrated how they navigate such dynamic interaction between the Prevent Duty and the socio-political context in which it is implemented.

In keeping with the incorporation of the Prevent Duty in a broader professional culture of safeguarding, the vast majority of respondents reported that they would pass on to their DSL anything they were unsure or uncomfortable about. For example, $86.9 \%$ of survey respondents 
stated they were likely or very likely to make a safeguarding query if a student draws or writes about weapons:

It depends on the child, but I would be concerned if they were obsessing over something like that [weapons]. I would raise it, even if it turned out to be nothing. (Anonymous survey respondent)

Although mentioning the need to observe children's behaviours over time and to be sensitive to the context of specific actions and statements, survey respondents reported that, in practice, they most often side-lined concerns, doubts, and ambiguities, in favour of referring:

It could be something or nothing but it is better to report and it be nothing that to ignore and it cause harm to someone. (Anonymous survey respondent)

In this context, time and again, falling back on wider safeguarding practices and policies, respondents reported that they would flag up any potential problems to allow the DSL to make the decision. Indeed, most of our respondents expressed trust in their schools' procedures, with $93.1 \%$ of survey respondents trusting their school to make sensible and appropriate decisions about referring pupils and staff to the Local Authority through the Prevent Duty. As a survey respondent put it:

I know I am well supported in school and I would report to DSL confidently, even if my concerns turned out to be wrong. (Anonymous survey respondent)

There was also broad agreement among survey respondents about the kinds of actions or behaviours that would prompt them to make a referral. Despite the fact that the majority had received training specific to this area, several survey respondents and interviewees mentioned 'common sense' as the main determinant for a referral. For example, when asked 'If you saw a student or staff member watching video clips of beheadings, would you make a safeguarding query?', the overwhelming majority of survey respondents $(87.5 \%)$ answered yes. They explained their choice by 
using emphatic exclamations such as: 'Does this need an explanation??!!! Of course!!', 'It is immoral!'; 'Definitely!', and 'Most definitely! That is completely unacceptable.' Only a handful of respondents shared more in-depth reflections on this issue, such as:

This is certainly a cause for concern but again does not instantly mean a student has been radicalised or has any intention of causing harm. It is important to find out why they sort [sic] out those videos and by who they had been told about the videos. It could be something as innocent as they had heard about it on social media and were curious about what people were talking about rather than seeking it out because they agreed with the video. (Anonymous survey respondent)

Most survey respondents (88\%) similarly described themselves as likely or very likely to make a safeguarding query if a student or staff member made hateful statements against an ethnic, sexual, or another minority group. In addition, $73.8 \%$ of survey respondents said that they were likely or very likely to make a safeguarding query if they heard a student or staff member express anger about immigration and non-British cultures. As one survey respondent put it:

this is most likely a racism concern and would need to be spoken to with DSL as it is very serious. (Anonymous survey respondent)

However, many pointed out that hateful statements and anger about immigration are possibly not a safeguarding concern, nor are they directly linked to radicalisation towards extremism. In this context, several survey respondents referred to different protocols when faced with potential instances of far-right extremism as compared with the red flags of Islamist extremism. In this vein, anti-immigration and hateful statements, in their views, would not result in referrals under the Prevent Duty:

I would think the person was racist and ignorant, but this doesn't necessarily mean they would be radicalised by a far-right group. (Anonymous survey respondent) 
Rather, these actions would be addressed through the school's equality and behaviour policy:

It would be dealt with following our behaviour policy. Parents would also be contacted and possibly invited in to discuss pupil monitored family early help. (Anonymous survey respondent)

The fact that the respondents were more likely to resort to Prevent in the case of actions commonly associated with Islamist extremism may be partly explained by the content of the training. Some interviewees reported that the training that they had attended had placed undue weight on Islamist extremism. As one respondent put it:

[the training] was way too orientated around so-called 'Muslim extremists' and it just had a little bit about white supremacy at the end, and it just was quite... perhaps biased? (E16, Assistant Headteacher, Birmingham)

In this context, one headteacher in Birmingham recounted a negative experience with training delivered by the police:

The initial training that we had was via the police... I invited a number of my parent governors, all the staff and I have quite a lot of staff that are from Muslim faith Muslim background and I asked everybody in school to attend really for this special training. I thought it was really important and we really [made] a big deal out of it and after the training it didn't go down as well as I would have liked. It was very instructional there was no debate there was no questions... The presentation we had was very one sided about we need to be aware that this could be happening within our schools and we need to look out for these signs and things like that. Actually there were parts of it that made me feel really uncomfortable with the audience that were sitting there. So I went away from that session and I went away wanting to do the WRAP training myself because I thought I'll get better informed and I'll do it myself in school. (E13, headteacher, Birmingham)

The Deputy Head in this same school also mentioned this event, observing that it 'made some members of the community feel uncomfortable' 
and 'was potentially a little bit inflammatory for some members of our team and community' (E15, Deputy Head, Birmingham).

However, as mentioned at the beginning of this section, the enactment of the Prevent Duty in primary schools is dynamic and influenced by the wider socio-political context. In this sense, survey respondents were largely aware of the increasing threat from right-wing extremism in the UK. $73.9 \%$ of them agreed or strongly agreed with the statement 'I worry that right-wing terrorist attacks will become more frequent'. In the free text comments, several respondents pointed at the rise of populist politics around Brexit, which were seen to have legitimised anti-immigration and hateful statements:

Islamophobia coupled with issues around immigration have to lead to a rise in right wing parties lashing out against communities that perceive to be less British /English than them. (Anonymous survey respondent)

One survey respondent also added that the changing political context may trigger right-wing extremism:

The popularity of parties such as Brexit and the emergence of right-wing groups in Europe give credence to right wing ideologies of a more extreme nature. (Anonymous survey respondent)

Thus, research participants pointed out that the changing political context affected the content of Prevent training and the enactment of the Duty at large. As one interviewee reflected:

Initially with Prevent, because of some of the things in the media and because it was more to do with Daesh and all of that, that's the only aspect schools focused on. What we didn't do is the far right, we didn't do any of those things. Now, I think sort of a year or two on we are broadening and looking at it in a wider view than perhaps initially. It was just because we had had significant things in the news and it was all around those sorts of things. (E3, Deputy DSL, Walsall) 
Another headteacher echoed this perspective in pointing out that the training 'has changed over the years so the first time it did seem to fall more on Islamic terrorism, but we feel that it is far more balanced now' (E6, Headteacher, Walsall).

When reflecting on raising a Prevent-related safeguarding query, research participants largely adopted a better-safe-than-sorry attitude, consistent with a wider professional culture of safeguarding. However, they also highlighted that the Prevent Duty was being enacted dynamically, responding to the wider socio-political context in the UK. As a consequence of this changing political landscape, for example, it was reported that Prevent training is placing more emphasis on right-wing extremism.

\section{Embedding the Prevent Duty into the Promotion of Fundamental British Values}

As already mentioned, most of our respondents did not problematise the incorporation of Prevent into wider safeguarding practices and, in fact, appeared to welcome Prevent as an extension of their duty of care. In the evidence collected through surveys and interviews, participants also spontaneously expressed support for the teaching of values in primary schools and for the embedding of Prevent into FBVs.

DfE guidelines state that 'schools and childcare providers can also build pupils' resilience to radicalisation by promoting fundamental British values' (DfE, 2015, p.5). Primary school educators in our study appear to have accepted these guidelines and incorporated them into their practice. During the interviews, when asked if the Prevent Duty is embedded in the curriculum, most interviewees brought up their school's commitment to FBVs:

In terms of the Prevent Duty as such, I mean, for us the document itself is only about three or four pages. So we looked at it and thought about what do we do already and what do we need to look at. You know, we knew that British values have to be taught, we were already doing some of it but, I mean, we just needed to make it much more clearer [sic]. I think there is a 
lot more of a sharper focus on what's being done now because of the Prevent Duty. I think people feel a responsibility. (E13, Headteacher, Birmingham)

Another interviewee provided a similar response:

So, the way that we kind of take it, is so much to the British values route. We at my school have this specific curriculum for our school called the Three Bs, erm, and it's about our school, and then Birmingham and Britain, so it's about being part of those three communities... when we looked at, erm, you know, different types of extremism and, erm, people, young people being drawn to that, we thought a lot about the isolation and actually we want our children to feel that they are part of our community so that they are not vulnerable to that kind of, erm, coercion as it were. (E16, Assistant Headteacher, Birmingham)

However, to make the most of their educational potential, some respondents believed that Prevent-related issues (as well as other issues such as FGM) should be tackled in a child-friendly way, being sensitive to the children's needs, and using age-appropriate terminology:

In terms of the curriculum we tend to do it in terms of how to keep safe as oppose to Prevent itself because of the age of the children. (E6, Headteacher, Walsall)

As a consequence, they reported embedding Prevent in the curriculum in subjects like religious education (RE) and Personal Social Health Education (PSHE), through topics such as 'relationship coercion', 'prejudice and discrimination', 'understanding different religions', 'be respectful of one another's beliefs', which are also discussed in school assemblies. As a headteacher described:

We don't stand in front of our children going 'right, today we're doing about Prevent and how you stop something that doesn't look right'. (E23, Headteacher, Birmingham)

Embedding Prevent into other subjects also helped solve practical challenges related with time and resources for the primary school curriculum: 
there are so many things at the moment that I feel particularly with the tight curriculum and particularly with the pressures placed on schools, particularly within inner city areas where let's be honest a lot of these problems do arise, you've got the same high expectations required, expected of these children and the teachers, so for example you know you've got to get a pass rate of $75 \%$ which is the same in my area of Birmingham as it is in leafy home counties you know it just seems a bit absurd. You've got this intensity of curriculum alongside a plethora of other problems, you know you've got social issues. So what I'd say is that whilst there's definitely will I just don't think there's the support or the time, the curriculum is incredibly tight and I feel as if you would need such a long time to address certain issues class by class and we just don't have that time and this is probably a well-trod point made by a lot of teachers but unfortunately this is the case. (E26, Teacher, Birmingham)

Throughout their interviews, participants displayed a positive acceptance of teaching values in primary education. Only one educator problematized the notion of FBVs, and the fact that at present it encompasses Prevent:

even calling it British values annoys me a little because surely some values are values to everybody. [...] You can almost alienate people by saying they're British but we talk about our values and what we hold dear here. (E13, Headteacher, Birmingham)

This positive acceptance of teaching values and normalisation of the label of FBVs contrasts with the findings of research in the early childhood context (Chap. 5), where educators are broadly supportive of teaching values, but critical of labelling them as 'British'.

\section{Conclusion}

In this chapter, we have examined the enactment of the Prevent Duty in primary schools in the West Midlands, focusing on the implementation of the Duty, the raising of Prevent-related safeguarding concerns, and on 
the embedding of Prevent into FBVs. We have reported three main findings.

First, we find that primary school educators display a positive acceptance of the Prevent Duty. One survey respondent summarised that Prevent is 'doing a great job and helping to educate people'. Similarly to secondary school teachers (see Chap. 7), primary school teachers in our study reported that Prevent has been successfully incorporated into wider safeguarding practices. Thus, despite being conscious of the need to observe children's behaviours over time and to be sensitive to the context of specific actions and statements, our respondents reported that they would rather be safe than sorry. This attitude is wholly consistent with wider safeguarding procedures and leads to educators raising safeguarding concerns and trusting that the school leadership will deal with them appropriately. In this context, very few respondents reflected on the potentially detrimental consequences of a Prevent referral for the children, their families, and the wider community.

Second, we found that the Prevent Duty is enacted dynamically, responding to the context of primary education but also to the changing wider socio-political context. For example, respondents reflected that, initially, the Prevent Duty was broadly perceived as focusing on instances of Islamist extremism. Our data shows that this emphasis has shifted and that educators and those responsible for training have started to pay more attention to right-wing extremism. This shift may reflect local circumstances: in 2018, the largest proportion of individuals 'who received Channel support for concerns related to right-wing extremism [...] was from the West Midlands' (Home Office, 2018, p.15). It is also likely to be a response to national efforts by the Home Office to underscore that Prevent addresses all forms of violent extremism. Perhaps most importantly, a large part of our data collection was carried out in the immediate aftermath of the Christchurch mosque shootings of March 2019, which placed right-wing political violence high on the news agenda.

Third, similarly to other practitioners, primary school educators in our study were broadly supportive of conveying values in their classrooms. In keeping with official guidance, the Prevent Duty is embedded in the primary school curriculum primarily through the teaching of FBVs. Educators reported that talking about FBVs helps tackle Prevent-related 
issues in a child-friendly way. Many interviewees expressed support for teaching values such as democracy and tolerance and viewed schools as best placed to do so. As a survey respondent summarised: 'In education we can educate pupils about the correct values for everyone without prejudice'. In fact, only one interviewee reflected on the potentially detrimental and alienating impact of labelling values as 'British'. The scarcity of such critical attitudes is in contrast to the findings reported in other chapters in this volume (see Chaps. 3, 5, and 8).

Finally, there is a clear need for more multi-method and multi-level research to test these findings in regions other than the West Midlands. However, one message emerged consistently from the survey and interviews: educators repeated time and again that if the Prevent Duty is to work in the interests of children and the wider community, the specific requirements and context of primary schools should be considered at all stages of its enactment—from training, to operationalisation, to referral.

\section{References}

Ball, S. J., Maguire, M., \& Braun, A. (2013). How schools do policy. Abingdon, UK: Routledge.

Bryan, H. (2017). Developing the political citizen: How teachers are navigating the statutory demands of the counter-terrorism and security act 2015 and the prevent duty. Education, citizenship and social justice, 12(3), 213-226.

Busher, J., Choudhury, T., \& Thomas, P. (2019). The enactment of the counterterrorism "prevent duty" in British schools and colleges: Beyond reluctant accommodation or straightforward policy acceptance. Critical Studies on Terrorism, 12(3), 440-462.

Davies, L. (2016). Security, extremism and education: Safeguarding or surveillance? British Journal of Educational Studies, 64(1), 1-19.

Department for Education. (2015). Prevent. Retrieved from https://assets.publishing.service.gov.uk/government/uploads/system/uploads/attachment_ data/file/439598/prevent-duty-departmental-advice-v6.pdf

Department for Education. (2019). Keeping children safe in education. Retrieved from www.gov.uk/government/publications/keeping-childrensafe-in-education $\% 2 \mathrm{D} \% 2 \mathrm{D} 2$ 
Elwick, A., \& Jerome, L. (2019). Balancing securitisation and education in schools: Teachers' agency in implementing the prevent duty. Journal of Beliefs \& Values, 40(3), 338-353.

Heath-Kelly, C., \& Strausz, E. (2019). The banality of counter-terrorism "after, after 9/11"? Perspectives on the prevent duty from the UK health care sector. Critical Studies on Terrorism, 12(1), 89-109.

Home Office. (2018). Individuals referred to and supported through the prevent programme, April 2017 to March 2018. Retrieved from https://assets.publishing.service.gov.uk/government/uploads/system/uploads/attachment_data/ file/763254/individuals-referred-supported-prevent-programme-apr2017mar2018-hosb3118.pdf

Home Office. (2019). Revised prevent duty guidance: For England and Wales. Retrieved from www.gov.uk/government/publications/prevent-duty-guidance/revised-prevent-duty-guidance-for-england-and-wales\#e-sector-specific-guidance

Jerome, L., Elwick, A., \& Kazim, R. (2019). The impact of the prevent duty on schools: A review of the evidence. British Educational Research Journal, 45(4), 821-837.

Mastroe, C. (2016). Evaluating CVE: Understanding the recent changes to the United Kingdom's implementation of prevent. Perspectives on Terrorism, 10(2), 50-60.

Ragazzi, F. (2017). Countering terrorism and radicalisation: Securitising social policy? Critical Social Policy, 37(2), 163-179.

Open Access This chapter is licensed under the terms of the Creative Commons Attribution 4.0 International License (http://creativecommons.org/licenses/ by/4.0/), which permits use, sharing, adaptation, distribution and reproduction in any medium or format, as long as you give appropriate credit to the original author(s) and the source, provide a link to the Creative Commons licence and indicate if changes were made.

The images or other third party material in this chapter are included in the chapter's Creative Commons licence, unless indicated otherwise in a credit line to the material. If material is not included in the chapter's Creative Commons licence and your intended use is not permitted by statutory regulation or exceeds the permitted use, you will need to obtain permission directly from the copyright holder.

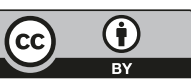

